\title{
Guía Práctica Clínica: Nueva guía de cuidados preventivos de la Fuerza de Tareas de Estados Unidos (primera entrega)
}

\begin{abstract}
Resumen
En 2001 la Fuerza del tareas Preventivas del EEUU (sigla en inglés: USPSTF) actualizó algunas recomendaciones de la segunda edición de su guía publicada en 1996 y algunas nuevas; lo que va constituyendo la tercera edición, a la que también se puede acceder gratuita y completamente en el sitio: http://www.ahrq.gov/clinic/uspstfix.htm

Ofrecemos aquí un resumen de las prácticas preventivas publicadas desde 2003 y su justificación clínica complementando la última entrega de EVIDENCIA que incluyó el material generado entre 2000 y 20032 . No hemos incluido las inmunizaciones y la profilaxis postexposición de enfermedades infecciosas pues ambas están regidas por las Normas Nacionales de Vacunación 2004 (http://www.direpi.vigia.org.ar/pdf/Normas_nacionales_03-2004.pdf).

En esta primera entrega resumimos las recomendaciones sobre consejería, indicación de suplementos y rastreo de problemas relacionados con estilos de vida. En una segunda entrega de EVIDENCIA nos explayaremos sobre las recomendaciones de rastreo del resto de las condiciones clínicas.
\end{abstract}

Ciapponi A. Nueva guía de cuidados preventivos de la Fuerza de Tareas de Estados Unidos (primera entrega). Evid actual pract ambul 2005;8:179-182.

\section{NUEVOS GRADOS DE EVIDENCIA}

Las modificaciones en la graduación de evidencia y el tipo de recomendación ya han sido comentadas previamente en esta revista. El cuadro 1, la tabla 1 y la figura 1 ofrecen un resumen de las mismas y remitimos al lector a dicho artículo.

Cuadro 1: grados de evidencia.

Buena: Se dispone de evidencia consistente obtenida de estudios bien diseñados y conducidos en poblaciones representativas con valoración directa de resultados.

Aceptable: La evidencia es suficiente pero limitada en cuanto al número, validez interna, externa o consistencia de los estudios; o bien valoración indirecta de resultados.

Pobre: La evidencia es insuficiente en cuanto al número o validez interna de los estudios, bien por brechas en la cadena de evidencia o falta de información sobre importantes resultados en salud.
Tabla 1: nueva nivel de recomendación de prácticas preventivas

\begin{tabular}{c|c|c|c|c}
\hline Tipo & Dirección & Evidencia & Resultados & Beneficio Neto \\
\hline A & Recomienda fuertemente & Buena & Mejoran & ++ \\
\hline B & Recomienda hacer & Aceptable & Mejoran & + \\
\hline C & No Recomienda (opcional) & Aceptable & Pueden mejorar & $" 0$ \\
\hline D & Recomienda no hacer & Aceptable & No mejoran & $0 \quad-$ \\
\hline I & No puede recomendar & Insuriciente & Indeterminados & $?$ \\
\hline
\end{tabular}

Figura 1: resumen de la nueva clasificación de las prácticas preventivas según la evidencia que la sustenta y la fuerza de la recomendación para realizarla.

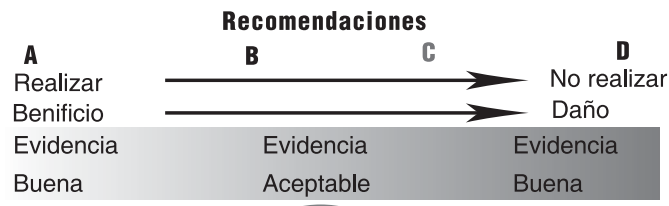

Evidencia

Tabla 1: resumen de las recomendaciones de consejería, indicación de suplementos y rastreo de problemas relacionados con estilos de vida.

\begin{tabular}{|c|c|c|c|c|}
\hline Problema & \multicolumn{2}{|c|}{ Práctica } & Recom. & Publ. \\
\hline Dolor de espalda & \multicolumn{2}{|c|}{ en adultos en atención primaria } & $\mathbf{I}^{1}$ & $2 / 2004$ \\
\hline Cáncer de piel & \multicolumn{2}{|c|}{ por médicos de atención primaria } & $\mathbf{I}^{2}$ & $10 / 2003$ \\
\hline Obesidad & \multicolumn{2}{|c|}{$\begin{array}{l}\text { de intensidad baja o media e intervenciones conductuales para descenso } \\
\text { sostenido de peso en adultos obesos o con sobrepeso }\end{array}$} & $\mathbf{l}^{3}$ & $12 / 2003$ \\
\hline \multirow[t]{2}{*}{ Lactancia } & \multicolumn{2}{|c|}{$\begin{array}{l}\text { de intervenciones conductuales y educacionales estructuradas de promo- } \\
\text { ción de la lactancia }\end{array}$} & $\mathbf{B}^{4}$ & \multirow[t]{2}{*}{$7 / 2003$} \\
\hline & \multicolumn{2}{|c|}{$\begin{array}{l}\text { de educación breve y consejo por médicos de atención primaria, consejo por } \\
\text { pares y materiales escritos }\end{array}$} & $I^{5}$ & \\
\hline \multirow[t]{2}{*}{ Abuso de alcohol } & \multirow{2}{*}{\multicolumn{2}{|c|}{$\begin{array}{l}\text { en adultos, incluso en embarazadas, en ámbitos de atención primaria } \\
\text { en adolescentes en ámbitos de atención primaria }\end{array}$}} & $\mathbf{B}^{6}$ & \multirow[t]{2}{*}{$4 / 2004$} \\
\hline & & & $\mathbf{I}^{7}$ & \\
\hline \multirow[t]{2}{*}{ Cese tabáquico } & \multicolumn{2}{|l|}{ en adultos y embarazadas } & $A^{8}$ & \multirow[t]{2}{*}{$11 / 2003$} \\
\hline & \multicolumn{2}{|l|}{ en niños y adolescentes } & $\mathbf{I}^{9}$ & \\
\hline Violencia familiar & \multicolumn{2}{|c|}{ niños, mujeres o ancianos (o sus cuidadores) } & $I^{10}$ & $3 / 2004$ \\
\hline Riesgo de suicidio & \multicolumn{2}{|c|}{ en población general por parte de médicos de atención primaria } & $I^{11}$ & $5 / 2004$ \\
\hline \multirow[t]{2}{*}{ Abuso de alcohol } & \multirow{2}{*}{\multicolumn{2}{|c|}{$\begin{array}{l}\text { en adultos, incluso en embarazadas, en ámbitos de atención primaria } \\
\text { en adolescentes en ámbitos de atención primaria }\end{array}$}} & $\mathbf{B}^{12}$ & \multirow[t]{2}{*}{$4 / 2004$} \\
\hline & & & $\mathbf{I}^{13}$ & \\
\hline \multirow{3}{*}{$\begin{array}{l}\text { Virus de la inmunodeficiencia } \\
\text { adquirida (VIH) }\end{array}$} & \multirow{3}{*}{$\begin{array}{l}\text { evaluar universalmente } \\
\text { conductas de riesgo } \\
\text { en embarazadas }\end{array}$} & Con riesgo: consejo y test & $\mathbf{A}^{14}$ & \multirow{3}{*}{$\begin{array}{l}\text { 7/2005 } \\
\text { Actualizado }\end{array}$} \\
\hline & & Sin riesgo & $\mathbf{C}^{15}$ & \\
\hline & & & $\mathbf{A}^{16}$ & \\
\hline \multirow[t]{2}{*}{ Vitaminas y antioxidantes } & \multicolumn{2}{|c|}{ para cáncer o enfermedad cardiovascular } & $I^{17}$ & \multirow[t]{2}{*}{$6 / 2003$} \\
\hline & \multicolumn{2}{|c|}{ beta-carotenos en prevención de cáncer o enfermedad cardiovascular } & $\mathbf{D}^{18}$ & \\
\hline Estrógenos y Progestágenos & \multicolumn{2}{|c|}{ para enfermedades crónicas en mujeres postmenopáusicas } & $\mathbf{D}^{19}$ & \multirow{2}{*}{$\begin{array}{l}5 / 2005 \\
\text { Actualizada }\end{array}$} \\
\hline Estrógenos & \multicolumn{2}{|c|}{$\begin{array}{l}\text { para enfermedades crónicas en mujeres postmenopáusicas histerec- } \\
\text { tomizadas }\end{array}$} & $\mathbf{D}^{20}$ & \\
\hline
\end{tabular}

* El grado de recomendación puede modificarse bajo ciertas circunstancias explicadas en el apartado correspondiente 
${ }^{1}$ Dolor de espalda en adultos (I)

No se hallo buena evidencia sobre ejercicios de fortalecimiento de espalda o modificación de factores de riesgo (aumento de actividad física, cese tabáquico o menor consumo de alcohol, etc.) para la prevención de lumbalgia en adultos. Evidencia limitada de que las sesiones educacionales en ámbitos laborales producen modestos beneficios a corto plazo respecto de individuos con dolor crónico o recurrente pero no hay evidencia sobre individuos saludables o de bajo riesgo de dolor de espalda. Algunas intervenciones como soportes mecánicos pueden aumentar el riesgo de dolor. No puede determinarse el equilibrio entere beneficios y daños de las distintas intervenciones preventivas del dolor de espalda.

${ }^{2}$ Cáncer de piel (I)

Evidencia insuficiente para determinar si el consejo médico ayuda a modificar conductas para reducir el riesgo de cáncer de piel. El consejo a padres puede incrementar el uso de pantallas protectoras en niños pero es insuficiente la evidencia sobre modificación de otras conductas (uso de ropas protectoras, reducción de la exposición solar o el uso de lámparas/camas solares o autoexamen de la piel) y sobre sus posibles daños.

${ }^{3}$ Intervenciones conductuales para el descenso sostenido de peso en adultos obesos o con sobrepeso (I)

Evidencia insuficiente para determinar si el consejo y/o las intervenciones conductuales ayudan al descenso sostenido de peso en adultos obesos y/o con sobrepeso (estudios de aceptable a buena calidad pero con resultados variados). No puede determinarse el equilibrio entre beneficios y daños potenciales de estas intervenciones.

${ }^{4}$ Intervenciones conductuales y educacionales estructuradas para la promoción de la lactancia (B).

Aceptable evidencia de que los programas que combinan educación y consejos conductuales se asocian a una mayor tasa de inicio y mantenimiento de la lactancia hasta los tres meses (material didáctico y al menos una sesión extendida seguida de protocolos estructurados con práctica y entrenamiento en habilidades y en resolución de problemas) y de que el apoyo mediante visitas personales o contactos telefónicos aumentan la proporción de mujeres que continúa con la lactancia hasta los seis meses (menor efecto protector que con programas educacionales). Pocos estudios evaluaron la combinación de ambas estrategias.

${ }^{5}$ Promoción de la lactancia: educación breve, consejo médico y por pares y materiales escritos (I)

No se halló evidencia en países industrializados de que el consejo de médicos de atención primaria y/o pares sea eficaz para promover la lactancia, como tampoco los materiales escritos usados como única estrategia.

${ }^{6}$ Abuso de alcohol en adultos en atención primaria (B).

Buena evidencia de que el rastreo en atención primaria puede identificar con exactitud a pacientes cuyo patrón de consumo no cumple los criterios de alcoholismo pero marca un riesgo aumentado de morbimortalidad; de que el consejo conductual breve con seguimiento reduce leve a moderadamente el consume de alcohol durante los siguientes seis a 12 meses; y de efectos positivos hasta cuatro o más años luego de la intervención. Limitada evidencia de que el rastreo y el consejo conductual reduzcan la morbilidad por alcohol.

Limitada evidencia de la efectividad del consejo para reducir el consumo de alcohol durante el embarazo. Estudios en población general adulta muestran que el consejo conductual es efectivo en mujeres que amamantan. Los beneficios del consejo conductual para reducir el abuso de alcohol superan los daños potenciales.

Consideraciones clínicas: Abuso de alcohol implica el consumo que pone en riesgo al individuo de problemas futuros. Se define como el consumo de más de siete medidas por semana o más de tres por ocasión para mujeres; y el de más de 14 medidas por semana o más de cuatro por ocasión para hombres.

EI AUDIT es la herramienta de rastreo más ampliamente utilizada en atención primaria pero el CAGE 4-ítems es el más popular en dicho ámbito. Las estrategias de rastreo deben ser seleccionadas según el ámbito y la población (disponibles en http://www.niaaa.nih. gov/publications/Practitioner/HelpingPatients.htm\#step1b)

Las intervenciones iniciales incluyen un consejo inicial de 15 minutos, feedback, consejo y fijar metas. La mayoría incluyen mayor asistencia, intervenciones motivacionales y seguimiento. El intervalo óptimo de rastreo es desconocido. Pacientes con pasado alcohólico, adultos jóvenes y otros grupos de riesgo (ej: fumadores) pueden beneficiarse de rastreos más frecuentes. Las embarazadas deben ser informadas de los efectos nocivos del alcohol sobre el feto y debe aconsejarse la abstinencia.

${ }^{7}$ Abuso de alcohol, en adolescentes en ámbitos de atención primaria (I)

El cuestionario CRAFFT fue recientemente validado para el rastreo de abuso de sustancias en adolescentes en atención primaria pero sus beneficios serán inciertos mientras no se evalúen intervenciones en este ámbito y para esta población. La evidencia en ámbitos de atención primaria es insuficiente como para valorar los potenciales beneficios y daños del rastreo, y el consejo conductual en adolescentes.

${ }^{8}$ Cese tabáquico, en adultos y embarazadas (A)

Hay buena evidencia de que intervenciones breves de cese antitabáquico en adultos, incluyendo rastreo, consejos conductuales breves (menos de tres minutos) y la farmacoterapia en ámbitos de atención primaria son efectivos, incrementando el número de personas que abandonan el tabaquismo y de abstinentes al año. Aunque la mayoría de los ensayos no proveen evidencias directas, hay buena evidencia de que el cese tabáquico reduce el riesgo de enfermedad coronaria, accidente cerebrovascular y enfermedad pulmonar.

Se concluyó que hay buena evidencia indirecta de que, aún pequeños aumentos de las tasas de abandono debidas al consejo, producirían importantes beneficios en salud por lo que los beneficios del consejo superan holgadamente los daños potenciales.

Hay buena evidencia de que las intervenciones extendidas de cese antitabáquico (cinco a 15 minutos) usando mensajes y materiales de autoayuda específicos para fumadoras embarazadas, comparadas con intervenciones breves genéricas, incrementan substancialmente la tasa de abstinencia durante el embarazo e incrementan el peso de los neonatos. Aunque las recaídas son muchas durante el posparto, se concluye que reducir el tabaquismo durante el embarazo tiene, probablemente, importantes beneficios en salud para el bebé y para la madre.

Se concluyó que los beneficios del consejo superan los daños potenciales.

${ }^{9}$ Cese tabáquico, en niños y adolescentes (I)

Se hallo limitada evidencia de que el rastreo y el consejo en niños y adolescentes en ámbitos de atención primaria sean efectivos previniendo el inicio o promoviendo el abandono del tabaco. No puede determinarse el balance entre beneficios y daños de las intervenciones preventivas o de cese antitabáquico en ámbitos clínicos en niños y adolescentes.

\section{${ }^{10}$ Violencia familiar en niños, mujeres o ancianos (I)}

No se encontraron evidencias directas de que rastrear violencia familiar reduzca la invalidez o la muerte prematura. Tampoco se encontraron estudios que determinen la exactitud de las herramientas de rastreo para identificar violencia familiar o de pareja entre los niños, mujeres o adultos mayores en la población general. Hay aceptable a buena evidencia de que las intervenciones reducen el daño a los niños cuando abuso del niño o el abandono se había evaluado. La evidencia es limitada acerca de si las intervenciones reducen el daño a las mujeres y ningún estudio que examinó la efectividad de intervenciones en los adultos mayores. Ningún estudio evaluó directamente los daños del rastrear y las 
intervenciones para violencia familiar. No puede determinarse el equilibrio entre los beneficios y lod daños de rastrear violencia familiar entre los niños, mujeres, o los adultos mayores.

Consideraciones clínicas: Todos los médicos que examinan niños y adultos debieran estar alertas a signos y síntomas físicos y de conducta asociados a abuso o abandono. Los pacientes deberían recibir documentación de sus hallazgos físico (ej. fotografías, mapas corporales) tratamiento para las lesiones físicas, el consejo por un profesional de salud mental experimentado, números de teléfono de centros de crisis locales y ser ayudados a obtener resguardo y protección.

Los factores asociados con abuso o abandono del niño incluyen: bajo ingreso, bajo nivel educativo materno, familia grande, baja edad maternal, padre único, perturbaciones psiquiátricas parentales y presencia de un padrastro. Los factores asociados a violencia con la pareja incluya: edad joven, bajo ingreso, embarazo, problemas mentales, o abuso de alcohol o drogas por parte de víctimas o perpetradores, estar separado o divorciado, e historia abuso sexual y/o físico en la niñez. Los factores asociados con el abuso de adultos mayores incluyen: la edad creciente, bajo ingreso, deterioro funcional, invalidez cognitiva, abuso de substancias, baja autoestima o estado emocional, convivencia, y falta de apoyo social.

Se han estudiado varios instrumentos para rastrear a los padres para el abuso del niño, pero su capacidad de predecir el abuso del niño o el abandono es limitado. También se han desarrollado instrumentos para rastrear violencia a la pareja pero ninguno se ha validado contra resultados mensurables. Sólo unos instrumentos de rastreo (CASE y HSEAST) se han desarrollado para identificar pacientes mayores víctimas potenciales de abuso 0 a sus cuidadores abusivos. Estas herramientas correlacionan bien con instrumentos previamente validados aplicados en la comunidad, pero no se han probado en atención primaria.

Los programas de visita domiciliaria a madres de alto riesgo (en base factores sociodemográficas) han mejorado los resultados de desarrollo infantil y disminuido la incidencia de abuso y abandono del niño, así como la proporción de actividad delictiva y de uso de drogas en la madre.

\section{${ }^{11}$ Riesgo de suicidio en población general por parte de médicos} de atención primaria (I)

No se encontró evidencia de que rastrear riesgo del suicidio reduce las tentativas de suicidio o la mortalidad. Hay evidencia limitada en la exactitud de las herramientas para identificar el riesgo del suicidio en atención primaria, incluso las herramientas de rastreo para identificar aquéllos de alto riesgo. Hay insuficiente evidencia de que el tratamiento de aquellos de alto riesgo reduzca las tentativas de suicidio o la mortalidad. No se encontraron estudios que hayan evaluado directamente los daños de rastrear y tratar pacientes en riesgo de suicidio. No puede determinarse el equilibrio de beneficios y daños de rastrear el riesgo de suicidio en atención primaria.

Consideraciones clínicas: los factores de riesgo más fuertes para el intento de suicidio incluyen: trastornos del humor u otros desórdenes mentales, abuso substancias, historia de autoagresión e historia de tentativas de suicidio. El riesgo del suicidio se evalúa a lo largo de un continuo que va desde la ideación suicida (relativamente menos severo) a la ideación suicida con un plan (más severo). Las mayoría de las prueba de rastreo del riesgo de suicidio no se validaron en atención primaria, salvo limitadas evaluaciones del SDDS-PC.

\footnotetext{
${ }^{12}$ Abuso de alcohol en adultos, incluso en embarazadas, en ámbitos de atención primaria (B)

Hay buena evidencia de que el rastreo en atención primaria puede identificar con exactitud a pacientes cuyo patrón de consumo no cumple los criterios de alcoholismo pero marca un riesgo aumentado de morbimortalidad. Hay buena evidencia de que el consejo conductual breve con seguimiento reduce leve a moderadamente el consume de alcohol por durante los siguientes seis a 12 meses. Hay evidencia de efectos positivos hasta cuatro o más años luego de la intervención pero hay limitada evi-
}

dencia de que el rastreo y el consejo conductual reduzcan la morbilidad por alcohol. La evidencia de la efectividad del consejo para reducir el consumo de alcohol durante el embarazo es limitada, sin embargo los estudios en población general adulta muestran que el consejo conductual es efectivo en mujeres que amamantan. Los beneficios del consejo conductual para reducir el abuso de alcohol superan los daños potenciales.

Consideraciones clínicas: abuso de alcohol implica el consumo que pone en riesgo al individuo de problemas futuros. Se define como más de siete medidas por semana o más de tres por ocasión en mujeres; y más de 14 medidas por semana o más de cuatro por ocasión para hombres. El AUDIT es la herramienta de rastreo más ampliamente utilizada en atención primaria pero el CAGE 4-ítems es el más popular en dicho ámbito y ha sido validado en portugués. Las estrategias de rastreo deben ser seleccionadas según el ámbito y la población (disponibles en http://www.niaaa.nih.gov/publications/Practitioner/HelpingPatients.htm\#step1b)

Las intervenciones iniciales incluyen un consejo inicial de 15 minutos, feedback, consejo posterior y fijación metas. La mayoría incluyen mayor asistencia, intervenciones motivacionales y seguimiento. El intervalo óptimo de rastreo es desconocido. Pacientes con pasado alcohólico, adultos jóvenes y otros grupos de riesgo (ej: fumadores) pueden beneficiarse de rastreos más frecuentes. Las embarazadas deben ser informadas de los efectos nocivos del alcohol sobre el feto, debiéndose aconsejar la abstinencia.

\section{${ }^{13}$ Abuso de alcohol en adolescentes en ámbitos de atención primaria (I)}

El cuestionario CRAFFT, ha sido recientemente validado para el rastreo de abuso de sustancias en adolescentes en atención primaria, pero sus beneficios serán inciertos mientras no se evalúen intervenciones en este ámbito y para esta población. La evidencia en ámbitos de atención primaria es insuficiente como para valorar los potenciales beneficios y daños del rastreo y el consejo conductual en adolescentes.

${ }^{14}$ Virus de la inmunodeficiencia adquirida, evaluación universal de conductas de riesgo y si las hay, consejo pretest seguido de rastreo $(A)$

Hay buena evidencia de que el las pruebas disponibles (la estándar o la rápida aprobada por la FDA) detectan VIH con precisión. Hay buena evidencia de que el tratamiento oportuno con el régimen HAART (sigla en inglés de tratamiento antirretroviral altamente activo) mejora los resultados de salud de los rastreados, incluyendo menor progresión y mortalidad. Dado que los falsos positivos son raros, los daños del rastreo son mínimos. Los daños potenciales por los falsos positivo son ansiedad, etiquetamiento y efectos en las personas cercanas. La mayoría de los efectos adversos del HAART, como trastornos metabólicos, pueden ser corregidos modificando el esquema o con tratamiento apropiado. Los beneficios de rastrear a individuos de riesgo superan sustancialmente los daños potenciales.

${ }^{15}$ Virus de la inmunodeficiencia adquirida $(\mathrm{VIH})$ en personas de bajo riesgo (C)

Hay aceptable evidencia de que el rastreo de adolescentes y adultos sin riesgo para VIH puede detectar casos adicionales y buena evidencia de que intervenciones oportunas, especialmente el HAART, mejorarían los resultados en salud. Sin embargo, el rédito de rastrear personas sin riesgo es bajo y existen daños potenciales ya mencionados. Los beneficios de rastrear de adolescentes y adultos sin riesgo para $\mathrm{VIH}$ son muy pequeños en relación al daño potencial como para justificar una recomendación general.

${ }^{16}$ Virus de la inmunodeficiencia adquirida (VIH) en embarazadas (A)

Hay buena evidencia para rastrear a embarazadas y aceptable, para el consejo prenatal universal y el testeo con pruebas estándares o rápidas en forma voluntarias, dado que incrementan el número de mujeres diagnosticadas y tratadas antes del parto. Hay buena evidencia de que el régimen HAART es aceptado por las 
embarazadas y de que reduce significativamente la tasa de transmisión madre-hijo sin evidencias de anomalías o problemas maternofetales (con la excepción de efavirenz). La detección temprana permite discutir la indicación de cesárea electiva y de evitar la lactancia materna para reducir la tasa de transmisión de $\mathrm{VIH}$. Los beneficios de rastrear a toda mujer embarazada superan sustancialmente los daños potenciales.

${ }^{17}$ Vitaminas y antioxidantes en prevención de cáncer o enfermedad cardiovascular (I)

Se encontró pobre evidencia como para determinar si el suplemento con vitaminas reduce el riesgo de enfermedad cardiovascular o de cáncer. La evidencia disponible de los ensayos aleatorizados es inadecuada o contradictoria y no puede determinarse la influencia de las variables confundidoras sobre los resultados observados en los estudios observacionales. No se puede determinar el equilibrio entre los beneficios y los daños del uso rutinario de suplementos de vitaminas $\mathrm{A}, \mathrm{C}$ o E; multivitamínicos con ácido fólico o combinaciones de antioxidantes para la prevención de cáncer o la enfermedad cardiovascular.

${ }^{19}$ Beta-carotenos en prevención de cáncer o enfermedad cardiovascular (D)

Se encontró buena evidencia de que los suplementos del betacaroteno no proporcionan beneficios en la prevención de cáncer o la enfermedad cardiovascular en adultos de mediana y mayor edad. En dos ensayos restringidos a fumadores pesados, los betacarotenos se asociaron con incidencias más altas de cáncer pulmonar y de mortalidad total. Se concluye que los beta-caroteno improbablemente proporcionen beneficios importantes y podrían causar daños en algunos grupos.

${ }^{20}$ Estrógenos y Progestágenos para la prevención de enfermedades crónicas en mujeres postmenopáusicas (D)

Se del encontró aceptable a buena evidencia de que la combinación de estrógenos y progestágenos tiene beneficios y daños.
Los beneficios incluyen menor riesgo de fracturas (buena evidencia) y menor riesgo de cáncer colorectal (aceptable evidencia). Los daños incluyen el mayor riesgo de cáncer de mama (buena evidencia) de tromboembolismo venoso (buena evidencia) de enfermedad coronaria (buena evidencia) de accidente cerebrovascular (aceptable evidencia) y de colecistitis (aceptable evidencia) demencia y peor función cognitiva (aceptable evidencia). La evidencia es escasa para determinar sus efectos sobre otros resultados importantes como cáncer ovárico, mortalidad por cáncer de mama, por enfermedad cardiovascular, o mortalidad total.

Se concluyó de que los efectos perjudiciales de estrógenos y progestágenos, probablemente excedan los beneficios en la prevención de enfermedades crónicas en la mayoría de las mujeres. No se evaluó la TRH para tratar síntomas menopáusicos, vasomotores (sofocos) o síntomas urogenitales. El balance de beneficios y daños para una mujer individual dependerá de sus preferencias personales, riesgos individuales para las enfermedades crónicas específicas, y la presencia de síntomas menopáusicos.

${ }^{20}$ Estrógenos para la prevención de enfermedades crónicas en mujeres postmenopáusicas histerectomizadas (D)

Se encontró buena evidencia de que el uso de estrógenos sin oposición tiene tanto beneficios como daños. Los beneficios probables incluyen menor riesgo de fracturas (buena evidencia). Los daños probables incluyen mayor riesgo de tromboembolismo venoso (aceptable evidencia) colecistitis, y accidente cerebrovascular (aceptable evidencia) demencia y peor función cognitiva (aceptable evidencia). En mujeres sin histerectomía, los estrógenos sin oposición aumentan el riesgo de cáncer endometrial. La evidencia es aceptable para determinar que los efectos de los estrógenos sin oposición no tienen beneficios sobre la enfermedad coronaria; y es insuficiente en cuanto al riesgo de cáncer de mama, colorectal y ovárico, o de la mortalidad por cáncer de mama y/o total. Se concluye que los efectos perjudiciales de los estrógenos sin oposición probablemente excedan a los beneficios en la prevención de enfermedades crónicas en la mayoría de las mujeres.

\section{Agustín Ciapponi [ Responsable del Área de Prevención de la Unidad de Medicina Familiar y Preventiva del Hospital Italiano de Buenos Aires. ]}

\section{Bibliografía Recomendada}

Guide to Clinical Preventive Services, Second Edition. 1996; Report of the U.S. Preventive Services Task Force.

Ciapponi A. Guía de cuidados preventivos de la USPSTF (3ra edición, 2000-2003). Evidencia en atención primaria 2003 Mayo-Junio Vol 6 Nro 3:79-84.

Ciapponi A. Guía de práctica clínica: Tercer reporte de la USPSTF. Evidencia en atención primaria 2001 Septiembre-OctubreVol 4 Nro 5:153-8.

Third Report of the Expert Panel on Detection, Evaluation, and Treatment of High Blood Cholesterol in Adults (Adult Treatment Panel III). http://www.nhlbi.nih.gov/guidelines/cholesterol/atp3full.pdf

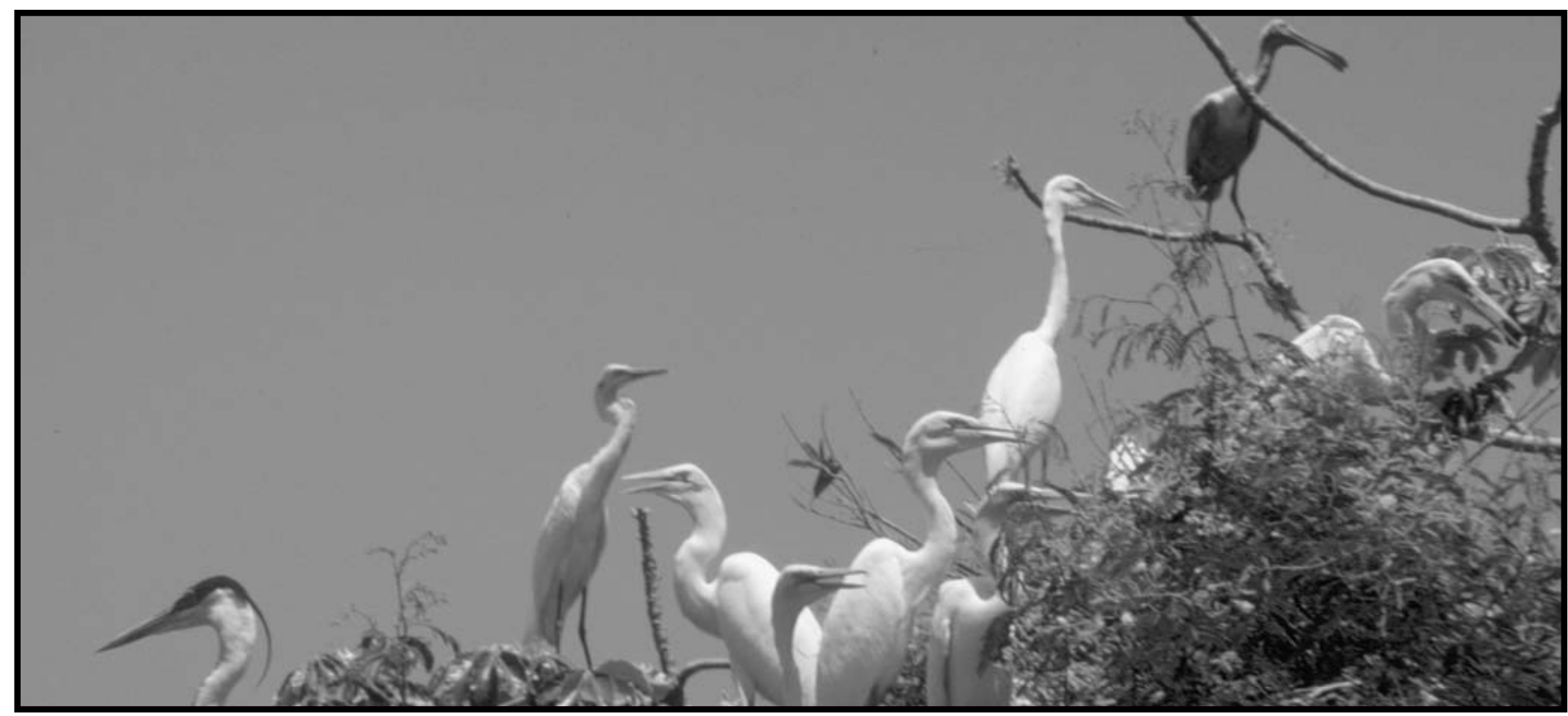

Gentileza Secretaria de Turismo de La Nación 